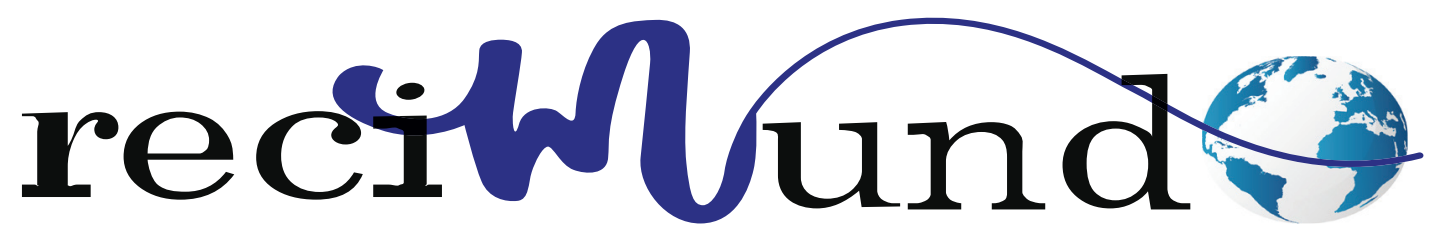

Revista Científica Mundo de la Investigación y el Conocimiento

DOI: 10.26820/recimundo/5.(2).abril.2021.26-36

URL: https://recimundo.com/index.php/es/article/view/1031

EDITORIAL: Saberes del Conocimiento

REVISTA: RECIMUNDO

ISSN: 2588-073X

TIPO DE INVESTIGACIÓN: Artículo de revisión

CÓDIGO UNESCO: 32 Ciencias Médicas

PAGINAS: 26-36

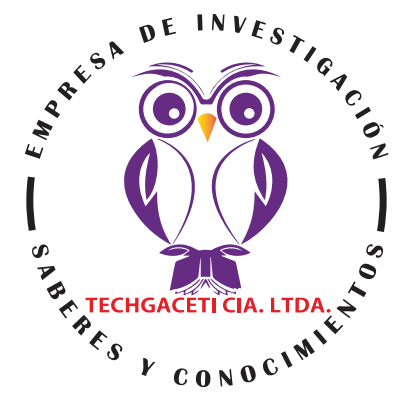

\title{
Consecuencias del embarazo de alto riesgo y su valoración fetal anteparto
}

Consequences of high-risk pregnancy and its antepartum fetal assessment

Conseqüências da gravidez de alto risco e sua avaliação fetal antepartum

Silvia Danixa Espinoza Moreira1; Jean Carlos Santos García²; Klever Javier Santos García3;

Maylen Andrea Zambrano Espinoza ${ }^{4}$

\section{RECIBIDO: 15/01/2021 ACEPTADO: 20/03/2021 PUBLICADO: 01/04/2021}

1. Médico Cirujano; Hospital Rafael Rodríguez Zambrano; Manta, Ecuador; silvia-danixa@hotmail.com; (iD https://orcid. org/0000-0002-4403-0223

2. Médico Cirujano; Hospital Rafael Rodríguez Zambrano; Manta, Ecuador; jean_28cs@hotmail.com; (iD https://orcid. org/0000-0001-6198-5833

3. Médico Cirujano; Hospital Rafael Rodríguez Zambrano; Manta, Ecuador; keisant6@hotmail.com; iD https://orcid.org/00000002-3754-3952

4. Médico Cirujano; Hospital Rafael Rodríguez Zambrano; Manta, Ecuador; maylenandrea_90@hotmail.com; (ID https://orcid. org/0000-0002-8269-2746

CORRESPONDENCIA

Silvia Danixa Espinoza Moreira

silvia-danixa@hotmail.com

Manta, Ecuador

() RECIMUNDO; Editorial Saberes del Conocimiento, 2021 


\section{RESUMEN}

Las complicaciones del embarazo y el parto son una de las principales causas de muerte y discapacidad entre las mujeres en edad reproductiva (15 a 44 años) de los países menos desarrollados. Además, están estrechamente asociadas a la mortalidad fetal y a condiciones precarias de salud y crecimiento para los niños. En la mayor parte de las gestaciones de riesgo, el momento más adecuado para iniciar el control del bienestar fetal se sitúa en torno a las 32-34 semanas. No obstante, en situaciones de riesgo muy elevado materno fetal -preeclampsia precoz, retraso del crecimiento intraute1ino precoz se puede iniciar en la semana 26-24. El monitoreo electrónico fetal anteparto consiste en la valoración de los latidos cardiacos fetales a través de un registro continuo de los latidos cardiacos fetales, a través de un monitor, quien se encarga de graficar la frecuencia cardiaca fetal por un tiempo no menor a 20 minutos, en el queda plasmado todas las características, que nos permite cuantificar los elementos de la frecuencia cardiaca fetal, que a la interpretación nos dará un diagnóstico. La metodología de la investigación es una revisión bibliográfica, apoyada en medios electrónicos, como fuente primaria de la obtención de la información. Son muchos los factores de riesgo a la que puede estar sometida una mujer embarazada, desde factores genéticos hasta factores de salud pre existentes como obesidad, diabetes, hipertensión, entre otras; que pueden convertir un embarazo en alto riesgo, y es así, ya que el nacimiento del feto, puede estar comprometido, dependiendo de riesgo que presente la mujer embarazada. Sin embargo, a pesar de estos riesgos, hay herramientas que pueden ayudar de una manera temprana y a tiempo a sobrellevar un embarazo de alto riesgo para que este pueda llegar a término, como es la valoración fetal anteparto por medio del monitoreo, que mide los valores de los latidos cardiacos fetales a través de un registro continuo y disminuir complicaciones tales como: convulsiones neonatales, parálisis cerebral o muerte fetal ante e intraparto, mientras disminuye la necesidad de intervenciones obstétricas innecesarias (parto vaginal instrumentado o cesárea).

Palabras clave: Anteparto, fetal, riesgo, embarazada, consecuencias.

\section{ABSTRACT}

Complications of pregnancy and childbirth are one of the leading causes of death and disability among women of reproductive age (15-44 years) in less developed countries. In addition, they are closely associated with fetal mortality and poor health and growth conditions for children. In most high-risk pregnancies, the best time to start monitoring fetal well-being is around 32-34 weeks. However, in situations of very high maternal-fetal risk - early pre-eclampsia, early intrauterine growth retardation, can begin at week 26-24. Antepartum electronic fetal monitoring consists of evaluating the fetal heartbeat through a continuous recording of the fetal heartbeat, through a monitor, which is in charge of graphing the fetal heart rate for a time not less than 20 minutes, In it, all the characteristics are captured, which allows us to quantify the elements of the fetal heart rate, which will give us a diagnosis upon interpretation. The research methodology is a bibliographic review, supported by electronic media, as the primary source for obtaining information. There are many risk factors that a pregnant woman may be subjected to, from genetic factors to pre-existing health factors such as obesity, diabetes, hypertension, among others; that can turn a pregnancy into high risk, and this is the case, since the birth of the fetus may be compromised, depending on the risk presented by the pregnant woman. However, despite these risks, there are tools that can help early and in time to cope with a high-risk pregnancy so that it can reach term, such as antepartum fetal evaluation through monitoring, which measures the values fetal heartbeat through continuous recording and reduce complications such as: neonatal seizures, cerebral palsy or fetal death before and intrapartum, while reducing the need for unnecessary obstetric interventions (instrumented vaginal delivery or cesarean section).

Keywords: Antepartum, fetal, risk, pregnant, consequences.

\section{RESUMO}

As complicações da gravidez e do parto são uma das principais causas de morte e incapacidade entre as mulheres em idade reprodutiva (15-44 anos) em países menos desenvolvidos. Além disso, estão intimamente associadas à mortalidade fetal e às más condições de saúde e crescimento das crianças. Na maioria das gravidezes de alto risco, o melhor momento para começar a monitorar o bem-estar fetal é por volta de 32-34 semanas. Entretanto, em situações de risco materno-fetal muito alto - pré-eclâmpsia precoce, retardo de crescimento intra-uterino precoce, pode começar na semana 26-24. 0 monitoramento eletrônico fetal antepartum consiste em avaliar o batimento cardíaco fetal através de um registro contínuo do batimento cardíaco fetal, através de um monitor, que se encarrega de graficar a freqüência cardíaca fetal por um tempo não inferior a 20 minutos, Nele são capturadas todas as características, o que nos permite quantificar os elementos da freqüência cardíaca fetal, o que nos dará um diagnóstico após a interpretação. A metodologia de pesquisa é uma revisão bibliográfica, apoiada por meios eletrônicos, como a principal fonte para a obtenção de informações. Há muitos fatores de risco aos quais uma mulher grávida pode estar sujeita, desde fatores genéticos até fatores de saúde pré-existentes como obesidade, diabetes, hipertensão, entre outros; que podem transformar uma gravidez em alto risco, e este é o caso, já que o nascimento do feto pode estar comprometido, dependendo do risco apresentado pela mulher grávida. Entretanto, apesar destes riscos, existem ferramentas que podem ajudar cedo e a tempo a lidar com uma gravidez de alto risco para que ela possa chegar a termo, como a avaliação fetal antepartum através do monitoramento, que mede os valores do batimento cardíaco fetal através do registro contínuo e reduz complicações como: convulsões neonatais, paralisia cerebral ou morte fetal antes e intra-parto, enquanto reduz a necessidade de intervenções obstétricas desnecessárias (parto vaginal instrumentado ou cesárea).

Palavras-chave: Antepartum, fetal, risco, gravidez, conseqüências. 


\section{Introducción}

Uno de los problemas más importantes a los que se enfrenta el obstetra y que compete a la práctica diaria de todas las instituciones que se dedican a la atención del parto, es el de conocer, y en todo momento, el "estado de bienestar del feto" entendiendo como este el equilibrio ácido-base fetal que nos garantice la preservación de una correcta función neurológica, y en definitiva, de todos los órganos fetales al nacimiento (Chango Sosa \& Velos Zurita, 2014).

Las complicaciones del embarazo y el parto son una de las principales causas de muerte y discapacidad entre las mujeres en edad reproductiva ( 15 a 44 años) de los países menos desarrollados. Además, están estrechamente asociadas a la mortalidad fetal y a condiciones precarias de salud y crecimiento para los niños, las intervenciones para prevenir y tratar las complicaciones del embarazo y el parto están bien documentadas, pero es necesario lograr un mayor compromiso y más inversiones para aumentar su disponibilidad (CABRERA CANSINO, 2018).

En la mayor parte de las gestaciones de riesgo, el momento más adecuado para iniciar el control del bienestar fetal se sitúa en torno a las 32-34 semanas. No obstante, en situaciones de riesgo muy elevado materno fetal -preeclampsia precoz, retraso del crecimiento intraute1ino precoz se puede iniciar en la semana 26-24 (Francés Ribera \& Terré Rull, 2014).

La evaluación del riesgo por sí sola, parece ser cada vez menos útil en la predicción de complicaciones en la gestación pues, aun sin importar los riesgos calculados, 90 a 95\% de los embarazos dan como resultado un recién nacido vivo y sano, lo cual contrasta con un gran número de óbitos fetales que ocurren en embarazos identificados como de bajo riesgo. A pesar de haber disminuido dramáticamente sus tasas de pre- sentación en los últimos 40 años, todavía se presenta gran dificultad en la evaluación y en la identificación de factores de riesgo, como obesidad, ser el primer parto, edad materna avanzada en la primera gestación y las gestaciones múltiples (Molina \& Alfonso, 2010).

Según la NOM-007-SSA2-1993 embarazo de alto riesgo es Aquel en el que se tiene la certeza o la probabilidad de estados patológicos o condiciones anormales concomitantes con la gestación y el parto, que aumentan los peligros para la salud de la madre o del producto, o bien, cuando la madre procede de un medio socioeconómico precario (Vergara, 2013).

La identificación del embarazo de alto riesgo distingue dos brazos principales:

- Alto riesgo obstétrico

- Alto riesgo fetal

Según la OMS La evaluación de los riesgos no es una medida única que se haga simplemente una sola vez, sino que es un procedimiento continuo durante todo el embarazo y el parto. En cualquier momento pueden aparecer complicaciones que pueden inducir a tomar la decisión de referir a la mujer a niveles atención más especializados (Vergara, 2013). 
Tabla 1. Guías clínicas para identificación de condiciones médicas durante el embarazo

\begin{tabular}{|c|c|}
\hline Condición medica & Descripción \\
\hline Cardiovascular & $\begin{array}{l}\text { Enfermedad cardiaca confirmada } \\
\text { Hipertensión }\end{array}$ \\
\hline Respiratoria & $\begin{array}{l}\text { Asma } \\
\text { Fibrosis quística }\end{array}$ \\
\hline Hematológica & $\begin{array}{l}\text { Hemoglobinopatías } \\
\text { Antecedente de tromboembolia } \\
\text { Trombocitocitopenia ( } \leq 100 ; 000) \\
\text { Enfermedad de Von Willebrand } \\
\text { Hemorragia } \\
\text { Isoinmunización materno-fetal }\end{array}$ \\
\hline Infeccioso & $\begin{array}{l}\text { HIV } \\
\text { Hepatitis } \\
\text { TORSCH } \\
\text { TB }\end{array}$ \\
\hline Inmunológicos & $\begin{array}{l}\text { LES } \\
\text { Escleroderma }\end{array}$ \\
\hline Endocrinos & $\begin{array}{l}\text { Hipertiroidismo/hipotiroidismo inestable } \\
\text { Diabetes }\end{array}$ \\
\hline Renal & Función anormal renal \\
\hline Neurológica & $\begin{array}{l}\text { Epilepsia } \\
\text { Miastenia gravis } \\
\text { Accidente cerebrovascular previo } \\
\text { Trastornos de la columna vertebral } \\
\text { Déficit neurológico } \\
\text { Fractura de pelvis }\end{array}$ \\
\hline Gastrointestinal & Pruebas funcionales hepáticas anormales, enfermedad de Crohn y colitis ulcerativa \\
\hline Psiquiátricas & $\begin{array}{l}\text { Depresión } \\
\text { Complicaciones en embarazos anteriores } \\
\text { Muerte fetal (óbito) o del neonato relacionada al parto } \\
\text { Encefalopatía hipóxico-isquémica } \\
\text { Preeclampsia/eclampsia/HELLP } \\
\text { Parto pretérmino } \\
\text { Ruptura uterina, incisión en ancla, cesárea corporal } \\
\text { Hemorragia post-parto primaria } \\
\text { Retención de placenta } \\
\text { Cesárea previa } \\
\text { Distocia de hombros }\end{array}$ \\
\hline Embarazo múltiple & $\begin{array}{l}\text { Placenta previa } \\
\text { EHAE } \\
\text { APP } \\
\text { RPM } \\
\text { DPPNI } \\
\text { Anemia }(\mathrm{Hb} \leq 8: 5 \mathrm{~g}=\mathrm{dL} \text { ) al inicio del parto } \\
\text { Óbito } \\
\text { Inducción del parto } \\
\text { Presentaciones de nalgas, situación transversa } \\
\text { IMC } \geq 35 \mathrm{Kg}=\mathrm{m} 2 \\
\text { Hemorragia recurrente anteparto } \\
\text { Feto pequeño (SGA/IUGR) } \\
\text { FCF anormal } \\
\text { Oligohidramnios/polihidramnios }\end{array}$ \\
\hline
\end{tabular}

Fuente: (Vergara, 2013). Elaboración propia. 
Se acepta generalmente que la patología de alteración del bienestar fetal se asocia con hipoxia del feto por nacer, la cual asocia a varias complicaciones obstétricas, afectando el nacimiento normal del intercambio gaseoso de feto y parturienta (Arias Matos, 2021).

El procedimiento diagnostico que estudia el comportamiento de la Frecuencia Cardiaca Fetal en relación con la vitalidad y movimientos del feto y las contracciones uterina se denomina Monitoreo Electrónico del feto, la cual nos advierte precozmente la hipoxia del feto, y podemos prevenir, alteración neurológica y consecuente fallecimiento del Recién Nacido (Arias Matos, 2021).

Respecto a la etiología, las causas del sufrimiento fetal son variadas y estas pueden agruparse en maternas, fetales y feto - placentarias. Dentro de las causas que pueden provocarla tenemos aquellas que determinan una disminución del aporte de sangre al útero en cantidad y calidad, como: la preeclampsia, la hipertensión, la diabetes, la anemia o los problemas pulmonares y las contracciones excesivas durante el trabajo

de parto o en partos prolongados. Los niveles de déficit de oxígeno pueden definirse del modo siguiente:

- Hipoxemia: definida por la disminución de la presión parcial de oxígeno (pO2).

- Hipoxia: indica que un órgano no recibe la suficiente cantidad de oxígeno.

- Asfixia: secundaria a una hipoxia intensa y/o de larga duración.

- Anoxia: término reservado para la falta total de oxígeno (Arias Matos, 2021).

\section{Metodología}

La metodología de la investigación es una revisión bibliográfica, ya que la misma se ha apuntalado en el uso de medios electrónicos para la obtención de la información de relevancia para la realización de este trabajo, ya que hay infinidad de autores que tocan la temática de estudio. El objetivo fundamental es hacer una síntesis lo más detallada posible, que sirva de ayuda para futuras investigaciones.

\section{Resultados}

Tabla 2. Factores del parto

\begin{tabular}{|c|l|}
\hline Factores & \multicolumn{1}{|c|}{ Descripción } \\
\hline $\begin{array}{c}\text { Inducción farmacológica del } \\
\text { parto }\end{array}$ & $\begin{array}{l}\text { Se lleva a cabo habitualmente en embarazadas patológicas por lo que } \\
\text { pueden surgir complicaciones más frecuentes que las que ocurrirán en } \\
\text { un parto normal. Las principales complicaciones son las que se derivan } \\
\text { de un exceso de dosis de oxitocina provocando hiperdinamias, } \\
\text { sufrimiento fetal, ruptura uterina hasta muerte fetal }\end{array}$ \\
\hline Embarazo prolongado & $\begin{array}{l}\text { Es aquel que sobre pasa el límite de } 294 \text { días o } 416 / 7 \text { semanas de } \\
\text { gestación. La mortalidad perinatal aumenta en proporción directa con la } \\
\text { prolongación de la gestación a las 42, reduplica a las 43 y se quintuplica } \\
\text { a las 44 semanas y se debe principalmente en un 30\% a insuficiencia } \\
\text { placentaria oligoamnios y un bajo nivel de reservas fetales que lleva a } \\
\text { sufrimiento fetal; otro porcentaje se debe a malformaciones del sistema } \\
\text { nervioso central. }\end{array}$ \\
\hline Circular de cordón & $\begin{array}{l}\text { La circular de cordón se puede dar en las partes fetales dando vueltas } \\
\text { en espira o circulares. Se presenta en un 25 -30 \% de los nacimientos } \\
\text { siendo más frecuente a nivel del cuello. En pocos casos por sí solos } \\
\text { puede ser causa de sufrimiento fetal agudo intraparto cuando esto } \\
\text { ocurre su instalación es lenta y siempre da tiempo de extraer al feto en } \\
\text { buenas condiciones }\end{array}$ \\
\hline Sufrimiento fetal & $\begin{array}{l}\text { Se define como una perturbación metabólica compleja debido a } \\
\text { disminución de los intercambios feto materno de evolución relativamente } \\
\text { rápida, que lleva a una alteración de la hemostasis fetal y que puede } \\
\text { provocar alteraciones tisulares irreparables o la muerte del feto. Las } \\
\text { causas de este pueden ser: Disminución del flujo sanguíneo uterino, } \\
\text { hipotensión, shock, reducción de la oxigenación sanguínea }\end{array}$ \\
\hline
\end{tabular}

Fuente: (Altamirano Delgado, 2008). Elaboración propia. 


\section{Monitoreo fetal anteparto}

El monitoreo electrónico fetal anteparto consiste en la valoración de los latidos cardiacos fetales a través de un registro continuo de los latidos cardiacos fetales, a través de un monitor, quien se encarga de graficar la frecuencia cardiaca fetal por un tiempo no menor a 20 minutos, en el queda plasmado todas las características, que nos permite cuantificar los elementos de la frecuencia cardiaca fetal, que a la interpretación nos dará un diagnóstico (Zorrilla Espinoza, 2020).

Esta prueba se basa en la evaluación del sistema nervioso, en caso de estar en buenas condiciones reaccionará con aceleraciones transitorias como respuesta a los movimientos fetales, el estudio tiene una especificidad del $90 \%$ en gestantes normales y en gestantes con patología llega hasta el $99 \%$, el problema de la prueba es su baja sensibilidad 45 a $50 \%$, por ello es necesario hacer otra prueba cuando este sale no reactivo (Zorrilla Espinoza, 2020).

La eficacia del monitoreo se juzga por su habilidad para disminuir complicaciones tales como: convulsiones neonatales, parálisis cerebral o muerte fetal ante e intraparto, mientras disminuye la necesidad de intervenciones obstétricas innecesarias (parto vaginal instrumentado o cesárea). No hay ensayos clínicos aleatorizados que comparen los beneficios de la monitorización de la FCF con ninguna forma de monitorización durante el trabajo de parto. Por tanto, los beneficios de la monitorización de la FCF son medidos a partir de informes que comparan auscultación intermitente con cardiotocografía (Valdivia Huamán, 2014).

Existen dos tipos de Monitoreo electrónico fetal: el Test no estresante (NST) y el Test estresante (TST).
El Test no estresante es un método de evaluación del estado de salud fetal durante el embarazo, basado en el estudio de las características de la frecuencia cardíaca fetal; en condiciones basales; tiene como objetivo fundamental la evaluación del estado de salud fetal durante el embarazo, identificando al feto que presumiblemente está sano y al feto que posiblemente pueda estar en situación comprometida, con el fin de en uno y otro caso se pueda corregir la situación antes de que se produzcan daños irreversibles en el feto (Valdivia Huamán, 2014).

Los criterios de valoración son los siguientes:

- Reactivo (Activo Reactivo): criterios de reactividad fetal presentes y normalidad de los parámetros de la frecuencia cardiaca fetal, es decir presenta al menos dos aceleraciones de 15 latidos/minuto, indicando bienestar del feto.

- No Reactivo (Activo no reactivo): criterios de reactividad ausentes y normalidad de la frecuencia cardiaca fetal, es decir que no presenta aceleraciones.

- Patológico (Hipoactivo): criterios de la reactividad ausentes y parámetros de la frecuencia cardiaca fetal alterados, quiere decir que existe aumento (taquicardia) o descenso (bradicardia) de la frecuencia cardiaca basal o desaceleraciones (Valdivia Huamán, 2014).

El Test estresante fue el primer test de reserva fetal propuesto a finales de los años 60 por Pose de la escuela de Montevideo, basado en la respuesta de la FCF a la presencia de contracciones inducidas (Valdivia Huamán, 2014).

Los criterios de valoración son los siguientes:

- TST Negativo: cuando en la gráfica no se observa desaceleraciones tardías ante la presencia de contracciones uterinas.

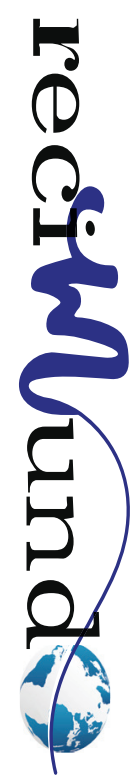


Usualmente, pero no necesariamente, se asocia con una buena variabilidad de la FCF y con presencia de ascensos de la FCF con los movimientos fetales.

- TST Positivo: cuando se observa en el $50 \%$ a más de desaceleraciones tardías en las contracciones uterinas registradas u observadas. Usualmente, pero no necesariamente, está asociada con una variabilidad de la FCF escasa y con ausencia de ascensos de la FCF con los movimientos fetales.

- TST Sospechoso no concluyente: cuando se observa en menos del $50 \%$ desaceleraciones tardías ocasionales en las contracciones uterinas registradas o las calificadas como desaceleraciones variables. La variabilidad de la FCF es normal o está disminuida y los ascensos de la FCF con los movimientos fetales pueden estar presentes.

- TST Insatisfactorio: Menos de tres contracciones en 10 minutos o un trazado que no es interpretable. Cuando no se logra obtener el patrón de contracciones uterinas empleando el máximo de oxitócica permitido (30mu). Este resultado puede ser debido a diversas circunstancias como, por ejemplo, obesidad de la paciente, polihidramnios, excesivos movimientos maternos o fetales e hipo fetal (Valdivia Huamán, 2014).

Tabla 3. Factores de riesgo para complicaciones durante el embarazo

\begin{tabular}{|c|c|}
\hline Factores & Observaciones \\
\hline Diabetes & $\begin{array}{l}\text { La diabetes mellitus manifiesta ocurre en } \geq 6 \% \text { de los embarazos, y la diabetes gestacional se } \\
\text { produce en aproximadamente el } 8,5 \% \text { de los embarazos. La incidencia está aumentando a medida } \\
\text { que crecen las cifras de obesidad. }\end{array}$ \\
\hline Pielonefritis & $\begin{array}{l}\text { Durante el embarazo, la bacteriuria recurrente es más frecuente y la incidencia de pielonefritis es } \\
\text { mayor. Veinte a } 35 \% \text { de las mujeres embarazadas con bacteriuria desarrollan una infección urinaria } \\
\text { (IU), y pueden experimentar pielonefritis. } \\
\text { La pielonefritis aumenta el riesgo de los siguientes: } \\
\text { - Rotura prematura de membranas. } \\
\text { - Trabajo de parto pretérmino. } \\
\text { - Síndrome de dificultad respiratoria del lactante. }\end{array}$ \\
\hline
\end{tabular}




\begin{tabular}{|c|c|}
\hline Edad materna & $\begin{array}{l}\text { Las adolescentes, que representan el } 13 \% \text { de todos los embarazos, tienen una mayor incidencia } \\
\text { de preeclampsia, trabajo de parto pretérmino y anemia, que a menudo conduce a una restricción del } \\
\text { crecimiento fetal. En las mujeres }>35 \text {, la incidencia de preeclampsia es mayor, al igual que } \\
\text { la diabetes gestacional, trabajo de parto disfuncional, abruptio placentae, fetos muertos y placenta } \\
\text { previa. Estas mujeres también tienen más probabilidades de trastornos preexistentes (p. } \\
\text { ej., hipertensión crónica, diabetes). Como el riesgo de anomalías cromosómicas fetales aumenta con } \\
\text { la edad materna, deben ofrecerse estudios genéticos. }\end{array}$ \\
\hline Peso materno & $\begin{array}{l}\text { Las mujeres con } \mathrm{IMC}<19,8 \mathrm{~kg} / \mathrm{m} 2 \text { antes del embarazo se consideran debajo del peso normal, lo } \\
\text { que predispone al bajo peso del neonato en el momento del nacimiento }(<2,5 \mathrm{~kg}) \text {. Estas mujeres } \\
\text { deben ser estimuladas a aumentar al menos } 12,5 \mathrm{~kg} \text { durante el embarazo. }\end{array}$ \\
\hline Talla materna & $\begin{array}{l}\text { Las mujeres de corta estatura }(<152 \mathrm{~cm}) \text { son más propensas a tener la pelvis pequeña, lo que puede } \\
\text { producir distocias con desproporción pelvicofetal o de hombro. Las mujeres bajas también son más } \\
\text { propensas a tener trabajos de parto prematuro y restricción del crecimiento intrauterino. }\end{array}$ \\
\hline Exposición a teratógenos & $\begin{array}{ll}\bullet & \text { Alcohol } \\
\bullet & \text { Tabaco } \\
\bullet & \text { Cocaína } \\
\bullet & \text { Algunos fármacos medicamentos que se venden con receta (véase Algunos } \\
& \text { fármacos con efectos adversos durante el embarazo). } \\
\text { La hipertermia o la exposición a temperaturas }>39^{\circ} \mathrm{C}(\mathrm{p} . \text { ej., en una sauna) durante el primer } \\
\text { trimestre se ha asociado con espina bífida. }\end{array}$ \\
\hline Exposición al mercurio & El mercurio en pescados y mariscos puede ser tóxico para el feto. \\
\hline Fetos muertos previos & $\begin{array}{l}\text { Haber tenido un feto muerto o un aborto tardío (es decir, entre las } 16 \text { y las } 20 \text { semanas) aumenta el } \\
\text { riesgo de muerte fetal en los embarazos posteriores. El grado de riesgo varía dependiendo de la } \\
\text { causa de una muerte fetal anterior. Se recomienda el control fetal usando pruebas anteparto (p. ej., } \\
\text { pruebas sin estrés, perfil biofísico). }\end{array}$ \\
\hline Parto pretérmino previo & $\begin{array}{l}\text { El parto pretérmino es el parto antes de las } 37 \text { semanas. Los partos pretérminos previos por trabajo } \\
\text { de parto prematuro aumentan el riesgo de partos pretérminos futuros; si un neonato pretérmino previo } \\
\text { peso }<1,5 \mathrm{~kg} \text {, el riesgo de parto pretérmino en el siguiente embarazo es del } 50 \% \text {. }\end{array}$ \\
\hline $\begin{array}{l}\text { Neonato previo con un } \\
\text { trastorno genético o } \\
\text { congénito }\end{array}$ & $\begin{array}{l}\text { El riesgo de tener un feto con un trastorno cromosómico aumenta en las parejas que ya han tenido } \\
\text { un feto o un neonato con un trastorno cromosómico (reconocido o no). La tasa de riesgo de recidivas } \\
\text { es desconocida para la mayoría de los trastornos genéticos. Muchas malformaciones congénitas son } \\
\text { multifactoriales; el riesgo de tener un feto posterior con malformaciones es de } \leq 1 \% \text {. }\end{array}$ \\
\hline $\begin{array}{l}\text { Embarazo multifetal } \\
\text { (múltiple) }\end{array}$ & 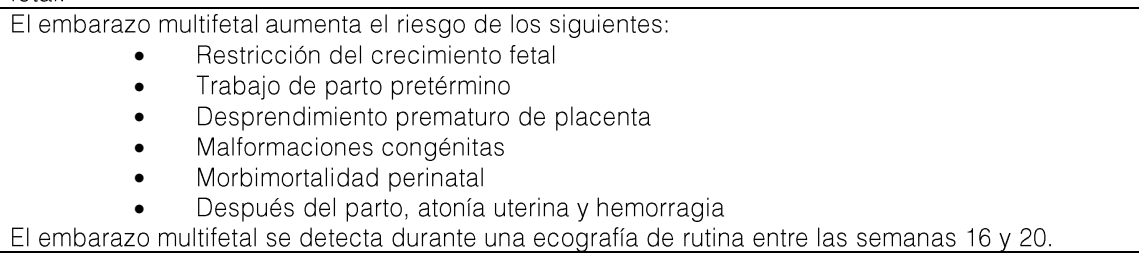 \\
\hline $\begin{array}{l}\text { Lesión previa durante el } \\
\text { nacimiento }\end{array}$ & $\begin{array}{l}\text { La mayor parte de las parálisis cerebrales y los trastornos del desarrollo neurológico se deben a } \\
\text { factores no relacionados con una lesión durante el parto. Lesiones como las del plexo braquial } \\
\text { pueden ser el resultado de procedimientos tales como forceps o vacuum extractor, pero con } \\
\text { frecuencia se originan en las fuerzas intrauterinas durante el trabajo de parto o una mala posición } \\
\text { durante las últimas semanas del embarazo. }\end{array}$ \\
\hline
\end{tabular}

Fuente: (Mittelmark, 2020). Elaboración Propia. 
Tabla 4. Consecuencias embarazo alto riesgo

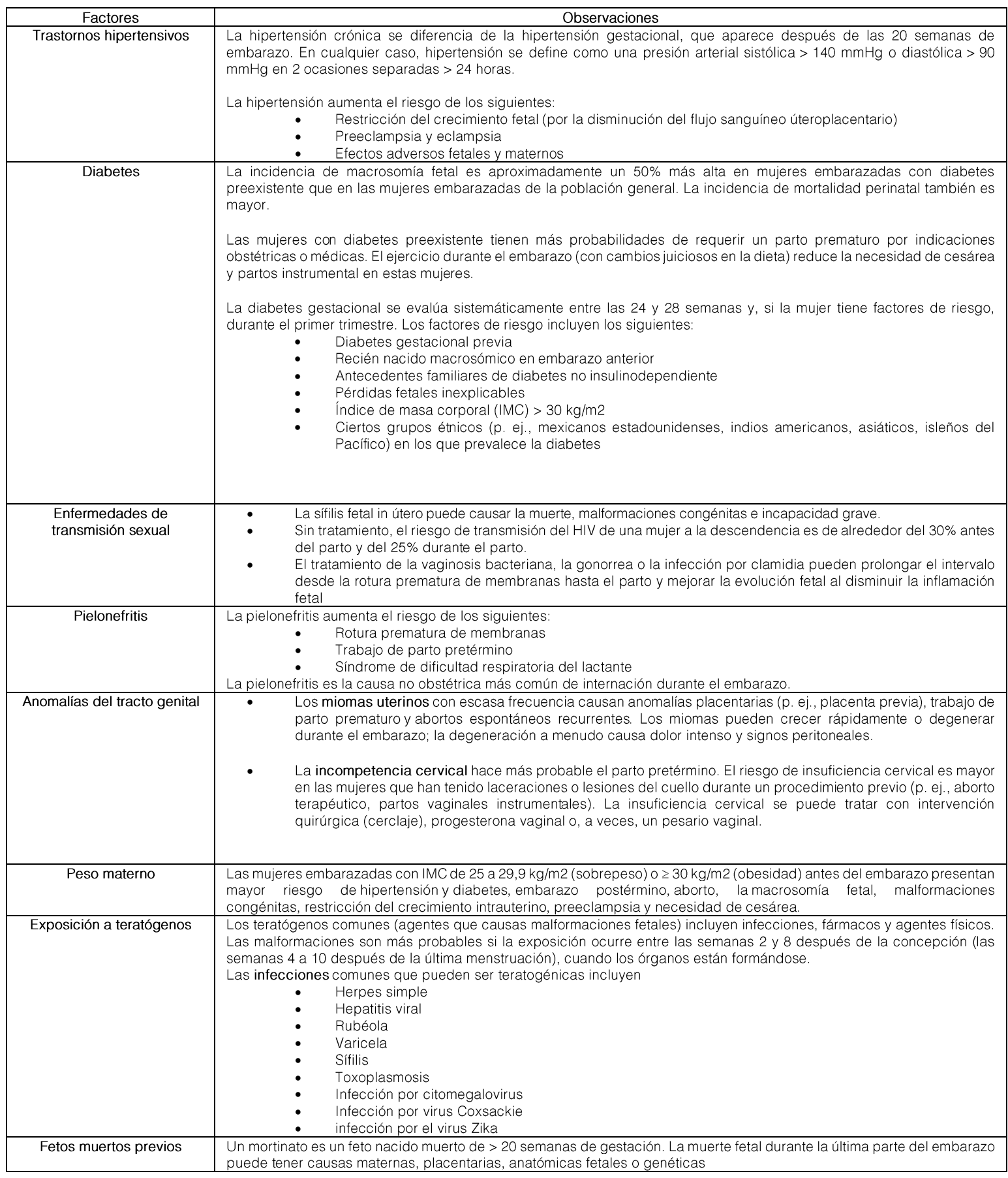

Fuente: (Mittelmark, 2020). Elaboración propia. 


\section{Conclusiones}

Son muchos los factores de riesgo a la que puede estar sometida una mujer embarazada, desde factores genéticos hasta factores de salud pre existentes como obesidad, diabetes, hipertensión, entre otras; que pueden convertir un embarazo en alto riesgo, y es así, ya que el nacimiento del feto, puede estar comprometido, dependiendo de riesgo que presente la mujer embarazada.

Sin embargo, a pesar de estos riesgos, hay herramientas que pueden ayudar de una manera temprana y a tiempo a sobrellevar un embarazo de alto riesgo para que este pueda llegar a término, como es la valoración fetal anteparto por medio del monitoreo, que mide los valores de los latidos cardiacos fetales a través de un registro continuo y disminuir complicaciones tales como: convulsiones neonatales, parálisis cerebral o muerte fetal ante e intraparto, mientras disminuye la necesidad de intervenciones obstétricas innecesarias (parto vaginal instrumentado o cesárea).

Las consecuencias fundamentales de un embarazo de alto riesgo, van direccionadas a como estas afectan el desarrollo del feto, y como son múltiples los factores que pueden incidir en un embarazo de alto riesgo, pues mayor es la posibilidad que un embarazo no llegue a término, un ejemplo de ello puede ser, las mujeres embarazadas obesas que pueden desarrollar una diabetes gestacional, una hipertensión arterial que puede desarrollar una preeclampsia, y que de no controlarse puede derivar en la interrupción del embarazo.

\section{Bibliografía}

Altamirano Delgado, R. X. (2008). Valoración de los principales factores de riesgo de óbito fetal registrados en el Hospital Juan Antonio Brenes Departamento de Madriz, 2005-2007.

Arias Matos, C. L. (2021). VALORACIÓN DEL MONITOREO ELECTRÓNICO FETAL ANTEPORTA EN EL DIAGNÓSTICO DE SUFRIMIENTO FETAL EN
PACIENTES CON GESTACIÓN MAYOR DE 37 SEMANAS EN EL HOSPITAL GENERAL DE OXAPAMPA-CERRO DE PASCO 2019.

CABRERA CANSINO, C. G. (2018). Monitoreo fetal anteparto para determinar la pérdida de bienestar fetal en gestantes con preeclampsia Hospital La Caleta julio-diciembre 2017.

Chango Sosa, P. E., \& Velos Zurita, A. (2014). Valor predictivo del monitoreo fetal anteparto para determinar complicaciones del neonato al nacimiento en mujeres embarazadas entre 18-35 años en la Unidad Metropolitana de Salud Sur de marzo-abril del 2014.

Francés Ribera, L., \& Terré Rull, C. (2014). Control del bienestar fetal: Monitorización biofísica anteparto. ROL. Revista Española de Enfermería, 37(12), 817-822.

Mittelmark, R. (09 de 2020). www.msdmanuals.com. Obtenido de https://www.msdmanuals.com/es-ve/ professional/ginecolog\%C3\%ADa-y-obstetricia/ embarazo-de-alto-riesgo/factores-de-riesgo-para-complicaciones-durante-el-embarazo

Molina, S., \& Alfonso, D. (2010). Muerte fetal anteparto: ¿ es una condición prevenible? Universitas Médica, 51(1), 59-73.

Valdivia Huamán, A. (2014). Eficacia del monitoreo electrónico anteparto en el diagnóstico de sufrimiento fetal -Instituto NacionalMaternoPerinatal-2013.

Vergara, M. A. (2013). Embarazo de alto riesgo.i Para quién?

Zorrilla Espinoza, J. M. (2020). MONITORIA FETAL ANTEPARTO EN EL HOSPITAL DANIEL ALCIDES CARRIÓN-CERRO DE PASCO, ENERO-FEBRERO 2020. 


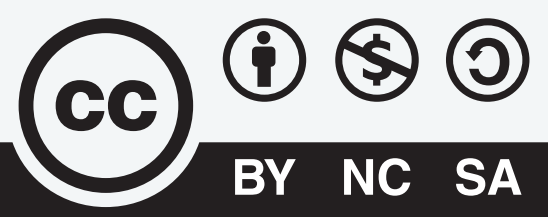

CREATIVE COMMONS RECONOCIMIENTO-NOCOMERCIAL-COMPARTIRIGUAL 4.0.

\section{CITAR ESTE ARTICULO:}

Espinoza Moreira, S. D., Santos García, J. C., Santos García, K. J., \& Zambrano Espinoza, M. A. (2021). Consecuencias del embarazo de alto riesgo y su valoración fetal anteparto. RECIMUNDO, 5(2), 26-36. https://doi.org/10.26820/ recimundo/5.(2).abril.2021.26-36 